\title{
Study of pervasiveness, antimicrobial vulnerability and resolution of best method for determining extended spectrum beta lactamases Escherichia coli isolates
}

\begin{abstract}
Introduction: The growing frequency of infections due to extended spectrum $\beta$-lactamase (ESBL) enzymes is a global dilemma, and the existence of ESBLs fluctuates between countries. Objective: To explored the occurrence of ESBLs producer E.coli in faecal carriage of
humans, antimicrobial susceptibility profile and their viable threat factors.

Method: For study 40 faecal samples were evaluated for antibiotic susceptibility profile and ESBLs production of all enterobacterial E. coli was phenotypically tested by the CLSI recommended methods.

Result: ESBL E. coli was prevalent among all E. coli isolates and its frequency was recorded to be $72.9 \%$. Current study revealed that ESBL producers $E$. coli or non ESBL producers $E$. coli both are highly susceptible to carbapenems and aminoglycosides class of antibiotics. Cefuroxime, cefaclor, and cefotaxime show high rate of resistivity followed by ampicillin, piperacillin, amoxicillin/clavulanic and amoxicillin/sulbactam in ESBLs as well as non ESBLs producers $E$. coli.
\end{abstract}

Conclusion: Higher antimicrobial resistivity was shown by ESBL producers E. coli than non ESBL producers E.coli.

Keywords: extended spectrum $\beta$-lactamases, E. coli, antimicrobial resistance, antibiotic
Volume 3 Issue 2 - 2019

\author{
Alka Mishra,' Renu Bist, ${ }^{2}$ Vinay Sharma ${ }^{3}$ \\ 'Department of Bioscience and Biotechnology, Banasthali \\ Vidyapith, India \\ ${ }^{2}$ Department of Zoology, Rajasthan University, India \\ ${ }^{3}$ Department of Life sciences, Amity University, India
}

\begin{abstract}
Correspondence: Renu Bist, Department of Zoology, Rajasthan University, Jaipur, Rajasthan, India, Tel 9174248 52622, Email renu_bisht22@yahoo.co.in
\end{abstract}

Received: January 26, 2019 | Published: March 26, 2019

\section{Introduction}

Gram negative bacteria (GNB) that have extended spectrum $\beta$-lactamase (ESBL) enzymes in clinical isolates have been documented as a most important public health challenge., ${ }^{1,2}$ $\beta$-lactamase enzyme are created by bacteria to hydrolyze the $\beta$-lactam ring in antibiotics, which confer resistance to bacteria and makes the drug treatment ineffective. ${ }^{3,4}$ ESBLs mediate bacterial resistance to $\beta$-lactam antibiotics having an oxyimino group (including third generation cephalosporins and aztreonam) and repressed by $\beta$-lactamase inhibitors, for example, clavulanic acid, tazobactam and sulbactam. ${ }^{5,6}$ ESBLs are expression products of plasmids and the genes which encoding these enzymes are moveable easily amongst different bacteria. ${ }^{7}$ Since 1983, after being first identified, they have been found globally. ESBLs exist in every region of the world and in most genera of enterobacteriaceae. These resistant isolates have been generally implicated in nosocomially obtained infections or outbreaks. ${ }^{8}$ Now ESBL producers are very frequent inside the community or in the wide healthcare settings. ${ }^{9}$ ESBLs are one of the major reasons of enhance in the incidence of colonization by multidrug resistance (MDR). The occurrence of MDR ESBLs in family Enterobacteriaceae is escalating swiftly and currently turns into a risk globally. ${ }^{10}$ Antibiotics uses generate a selective stress in the large intestine on host bacteria, mainly to the emergence of antimicrobial resistant organism, which in turn originate a raise in the quantity of carriers docking resistant bacteria and enhance the chance for bacterial infections. ${ }^{11}$ ESBL producing GNB are thought to be a crucial reason for the failure cephalosporin therapy. ${ }^{12,13}$ ESBL encoding genes that are mainly plasmid encoded comprise mostly TEM, SHV and CTX-M gene types. ${ }^{14,15}$ Enterobacteriaceae, such as Klebsiella pneumonia and Escherichia coli, are the primary ESBL producers. They confer bacterial resistance to penicillin and cephalosporin families that are common antimicrobial drugs. ${ }^{16}$ The colonization of ESBLs producer E. coli has been documented in both human being and animals and their prevalence increased drastically worldwide. ${ }^{17}$ Greater than 200 kinds of ESBLs were defined in various species of the family enterobacteriaceae and other non enteric organisms, with Pseudomonas aeruginosa and Acinetobacter genus. These organisms make variants of the TEM, SHV and CTX-M $\beta$-lactamases. ${ }^{18}$ Manifold variants of all gene are created with the aid of changing the configuration of amino acids within the $\beta$-lactamase active position. ${ }^{19,20}$

Carbapenems have been regard as the treatment alternative for severe infections as a result of ESBL producers. The mounting worldwide occurrence of ESBL linked infections is driving increased use of carbapenems drugs group, leading to choice stress for carbapenem resistance. ${ }^{8}$ ESBL resistance genes are specifically carried by plasmids. Plasmids may also convey genes encoding resistance to additional antibiotic class, including ampicillin $\mathrm{A}$, ampicillin $\mathrm{C}$, floroquinolone aminoglycosides, macrolides and chloramphenicol. Consequently, choice of treatment is confined for GNB that produce ESBLs due to the multiple resistance genes encoded within the plasmids. 
Since, there is an increasing threat of MDR bacteria, it is cardinal to execute a program which detects and reports ESBLs as fraction of their infection manage to limit the failure therapy caused by ESBL producing bacteria. ${ }^{21}$ Therefore, current investigation deals with phenotypic and biochemical method to noticed and affirm antibacterial resistance due to ESBL producing bacteria.

\section{Material and methods}

\section{Chemicals}

MacConkey agar (MA), nutrient agar (NA), eiosin methylene blue (EMB) agar, Kovak's reagent, methyl red, all antibiotics amikacin, ampicillin, ampicillin sulbactam, amoxiclave/clavunate, amoxicillin/sulbactam, aztreonam, cefaclor, cefotaxime cefuroxime, cefepime, ceftazidime, ciprofloxacin, co-trimaxazole, gentamicin, imipenem, levofloxacin, meropenem, netellin norfloxacin, ofloxacin, pipperacillin, pipperacillin/tazobactam, sparfloxacin, tetracycline, tobramicin and E-test strips under the study were purchased from Himedia (Mumbai, India).

\section{Sample collection}

Stool samples from patients who were on antibiotic dosages, were received in the Microbiology Laboratory of Escort Fortis Hospital, Rajasthan (India).

\section{Processing of collected samples and selection of E. coli}

Faecal samples were streaked on MA, NA and EMB agar. These petri plates were incubated aerobically for overnight at $37^{\circ} \mathrm{C}$ and they were observed for bacterial growth from obtained colonies and GNB were selected for study.

Morphological identification: GNB were identified on the basis of their morphological characteristics. Morphological study of bacterial colonies included colony shape, size, colour, elevation, margin, edges and Gram's staining attributes. ${ }^{22}$

Biochemical screening: For the determination of colony characters various biochemical tests were performed such as catalase test, oxidase test, indole test, methyl red test, triple sugar iron test, Simmon's Citrate Agar test, urease test and motility agar test. All biochemical tests were done in accordance to standard laboratory methods of identification for separation of $E$. coli isolates for the study. ${ }^{23}$

\section{Antibiotic susceptibility testing}

Antibiotic susceptibility testing was carried out using the Kirby Bauer disc diffusion method. Picked Three to five isolated E. coli colonies from the pure culture and suspended in $2-3 \mathrm{ml}$ of sterile peptone water in a test tube. The suspension turbidity was set to $0.5 \mathrm{Mc}$ Farkland standards. The test organisms were inoculated on the surface of Muller Hinton agar (MHA) with sterile cotton swab by lawn culture method. The inoculated plate was left for 10 to 15 minutes and placed the discs on the surface of petriplate through a disc dispenser with gentle pressure. The discs were located evenly in such a way so that they were $15 \mathrm{~mm}$ away from the rim of the Petridish and the gap between the centres of the two discs was around $24 \mathrm{~mm}$. Then plate were incubated at $37^{\circ} \mathrm{C}$ for 16 to 18 hours. ${ }^{23}$

\section{Screening of ESBL producing E. coli isolates}

ESBL production was confirmed using the CLSI recommendations for ESBL screening and confirmation tests as described below.
Screening of ESBL $\boldsymbol{E}$. coli isolates on Chromogenic agar: The screening of ESBL producer E. coli isolates was done by using HiCrome ESBL Agar Base according to manufacturer. HiCrome ESBL Agar Base is chromogenic screening medium for the selective isolation of ESBL producing organisms. Chromogenic mixture is used to differentiate the ESBL producing organisms on the basis of colour. Prepare the media by autoclaving at $15 \mathrm{lbs}$ pressure $\left(121^{\circ} \mathrm{C}\right)$ for 15 minutes. Then cool to $50^{\circ} \mathrm{C}$ and add two vials of HiCrome ESBL Selective Supplement (FD278). Mix well and transfer into sterilized Petri dish. Prepare the liquid suspension of $0.5 \mathrm{McF}$ arland from stool samples or from pure isolated colony. Then streak on the surface of petriplate and incubate aerobically at $37^{\circ} \mathrm{C}$ for overnight. ESBL producer $E$. coli produces pink or purple colour colonies. ${ }^{24}$

Double disc synergy test (DDST): Prepare a lawn culture by isolated bacteria on the surface of MHA. DDST was performed by placing a disk of amoxiclav/clavulanic acid $(20 / 10 \mu \mathrm{g})$, third generation cephalosporin $(30 \mu \mathrm{g})$ and aztreonam $(30 \mu \mathrm{g})$ were placed accordance to CLSI guidelines for anti microbial susceptibility test. ${ }^{25}$

Phenotypic confirmatory disc diffusion test (PCDDT): The probable ESBLs E. coli isolates were confirmed for the production of ESBL by phenotypic confirmatory disc diffusion test (PCDDT) as CLSI guidelines recommended for antimicrobial vulnerability tests. The antibiotics ceftazidime $(30 \mu \mathrm{g})$ and cefotaxime $(30 \mu \mathrm{g})$, alone and in combination with clavulanic acid $(10 \mu \mathrm{g})$ (beta-lactamase inhibitor) were used. ${ }^{26}$

E-test: In current study two types of E-test strips were used for the detection of ESBLs E. coli. ${ }^{27}$ E-test strips are porous paper drug saturated strips. These E-test strips are generated a constant concentration gradient of ceftazidime/ cefotaxime-clavulanate (mix/ $\mathrm{mix}+)(\mathrm{MIC}$ test range, $0.125-4 \mathrm{mcg} / \mathrm{ml})$ on one end and the remaining end generates a gradient of ceftazidime/ cefotaxime (MIC range, .125 to $16 \mathrm{mcg} / \mathrm{ml}$ ) plus $4 \mathrm{mcg} / \mathrm{ml}$ clavulanic acid. Similarly recently introduced cefepime/cefepime-clavulanate $(\mathrm{cpm} / \mathrm{cpm}+)$ Etest ESBL strip have cefepime (MIC test range, $0.25-16 \mathrm{mcg} / \mathrm{ml}$ ) and cefepime (MIC range, $0.064-4 \mathrm{mcg} / \mathrm{ml}$ ) plus $4 \mathrm{mcg} / \mathrm{ml}$ clavulanic acid. E-test strip technique, psychiatry, and their reading were carrying out accordance to the manufacturer's directives.

The significant of finding ESBLs producer E. coli was compared with non ESBLs producers by the means of Chi square test (SPSS 20). $P$ value was evaluated and regarded as significant when it was $>0.05$.

\section{Results}

Current investigation involved screening of a total of 40 samples for revealing ESBL E. coli.

E. coli ATCC 25922 and K. pneumoniae ATCC 700603 strains were used as negative and positive control.

\section{Morphological screening (Table I \& Figure IA-IC)}

Out of 40 stool samples studied, a total of 37 isolates of E. coli were identified on the basis of their morphological traits. They were pink in colour, rod shaped (individually) yet in circular colonies, Colony is pink on MA due to lactose fermentation, whereas in NA white colonies are obtained. EMB agar produced a distinctive metallic green sheen on E.coli colonies. They are circular, Small (2-3mm in diameter), convex, smooth, with entire margin and translucent and non mucoid. 
Table I Colony morphology and staining attributes of E. coli revealed in stool samples

\begin{tabular}{lll}
\hline S. No & Characteristics & Observation \\
\hline I & Shape & Circular \\
2 & Size & Small; 2-3mm diameter \\
3 & Colour & Pink (lactose fermenting) in MA, white \\
& in NA and metallic green in EMB agar \\
4 & Elevation & Convex \\
5 & Margin & Entire \\
6 & Edges & Smooth \\
7 & Light transmittance & Translucent \\
8 & Gram's stain & Gm-ve \\
\hline
\end{tabular}

EMB, eiosin methylene blue agar; MA, MacConkey agar; NA, nutrient agar

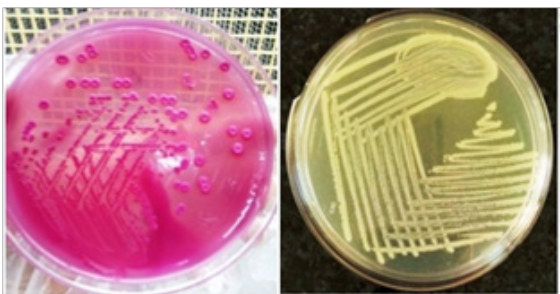

(a)

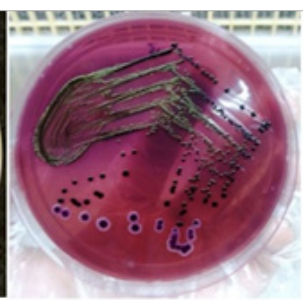

(c)

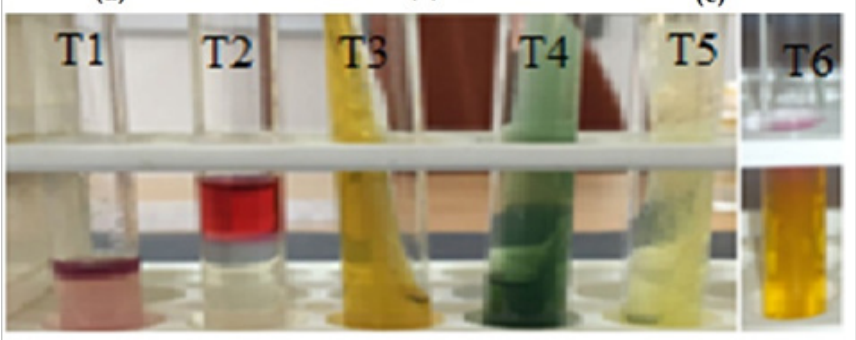

(d)

Figure I Morphological attributes and of $E$. coli colonies grown over (A) MacConkey agar, (B) nutrient agar (C) eosin methylene blue agar and (D) biochemical reactions of $E$. coli isolates.

$\mathrm{A} / \mathrm{A}$, Acidic/acidic; $\mathrm{TI}$, indole test; $\mathrm{T} 2$, methyle red test; $\mathrm{T} 3$, triple suger iron test; T4, simmon citrate agar test; T5, urease test; T6, mannitol motility agar test

\section{Biochemical tests (Figure ID)}

In biochemical screening $E$. coli isolates showed positive reaction for catalase, indole, and methyl red, whereas negative response recorded in the case of oxidase. E. coli isolates with triple sugar iron test showed gas production (A/A). They showed negative reaction for Simmons citrate agar and urease enzyme and motile in mannitol motility agar.

\section{Antibiotic sensitivity testing (Figure 2)}

Current study revealed that ESBL producers E. coli or non ESBL producers $E$. coli both are highly susceptible to imipenem $83.7 \%$, meropenem $94.5 \%$ and aminoglycosides $94.5 \%$ group of antibiotics. Cefuroxime, cefaclor, and cefotaxime show high rate of resistivity followed by ampicillin, piperacillin, amoxicillin/clavulanic and amoxicillin/sulbactam in ESBLs as well as non ESBLs producers $E$. coli. Whereas resistivity pattern towards ceftazidime and cefipime was
$48.6 \%$ and $51.3 \%$. Floroquinolones, trimethoprim/sulfamethoxazole, tetracycline are moderate sensitive for all $E$. coli isolates between 32 to $54 \%$. Susceptibility to other $\beta$-lactam inhibitors is good between 70 to $78 \%$. E. coli isolates from stool sample showed susceptibility to third generation cephalosporin was further decreased. ESBLs producers showed homogeneity in the resistance pattern, which were multi drugs resistant with at least a resistance to 16 antibiotics out of the 25 tested. E. coli showing higher resistance to cefotaxime $92.5 \%$ in ESBL producers and 30\% in non ESBL producers compared to ceftazidime $51.8 \%$ in ESBL producers and $10 \%$ in non ESBL producers. Higher resistance was shown by ESBL producers than non ESBL producers.

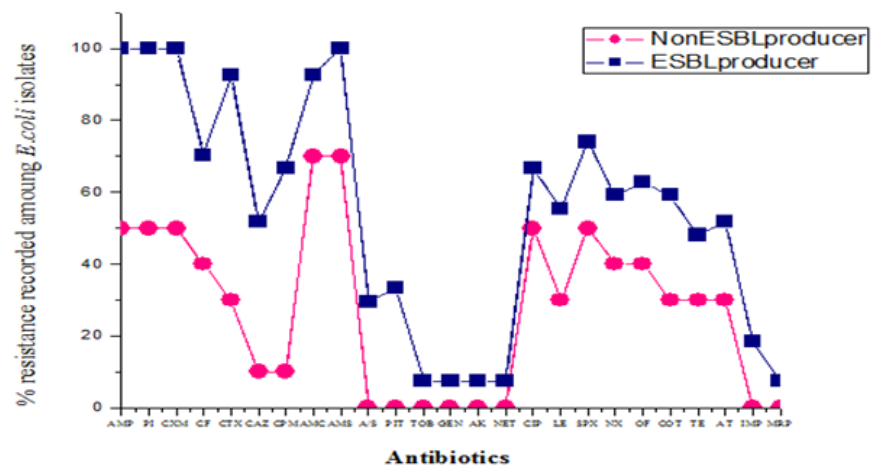

Figure 2 Diagram exhibiting a comparison of antibiotic resistance between ESBL producers and non ESBL producers.

AMP, ampicillin;AT, azeotranum; PI, pipperacillin; CXM, cefuroxime; CF, cefaclor CTX, cefotaxime; CAZ, ceftazidime; CPM, cefepime; AMC, amoxicillin/ clavunate;AMS, amoxicillin/sulbactam;A/S, ampicillin/sulbactam; PIT, piperacillin/ tazobactam; TOB, tobramicin; GEN, gentamicin; AK, amikacin; NET, netilmicin; CIP, ciprofloxacin; LE, levofloxacin; SPX, sparfloxacin; NX, norfloxacin; OF, ofloxacin; COT, co-trimoxazole; TE, tetracycline; IMP, imipenem; MRP, meropenem

\section{Confirmation of ESBL producers}

Confirmation using chromogenic agar (Figure 3): Pink to burgundy, translucent colonies were produced on ESBL screen chromogenic agar. We revealed $67.5 \%$ E. coli isolates as ESBLs producers by using Hichrome ESBL agar base.

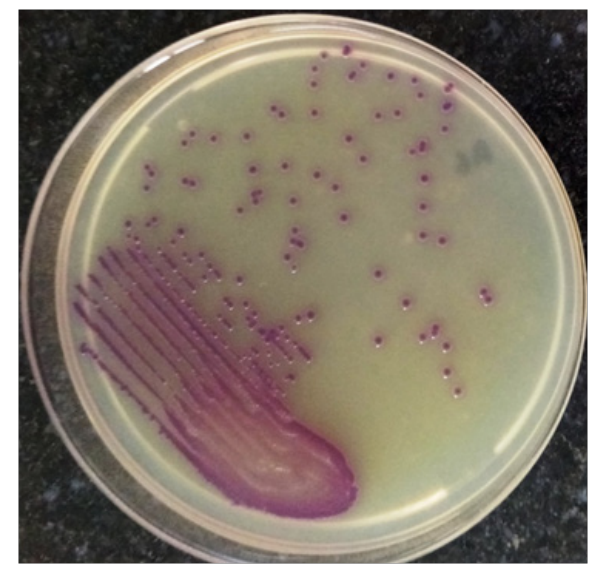

Figure 3 ESBL E. coli colonies on HiChrome ESBL agar base.

DDST (Figure 4A \& 4B): In DDST, Out Of the $37 \mathrm{E}$. coli isolates screened for ESBL production, by DDST, ESBLs positive occurrence rate was revealed to be $48.6 \%$. 
PCDDT (Figure 4C \& 4D): The overall prevalence of ESBLS producer E. coli was revealed to be $72.9 \%$ by PCDDT. Out of 27 ESBLs producer isolates 17 showed positive results with ceftazidime and cefotaxime and 8 showed positive tests with only cefotaxime and remaining 2 with ceftazidime.

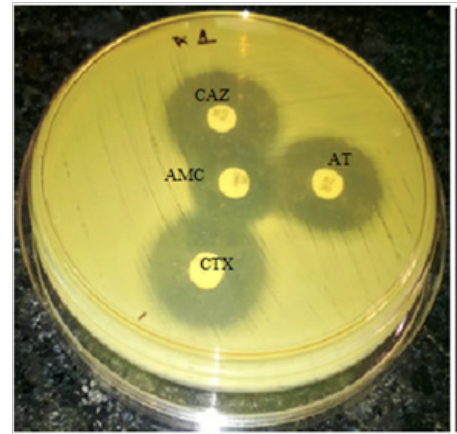

(a)

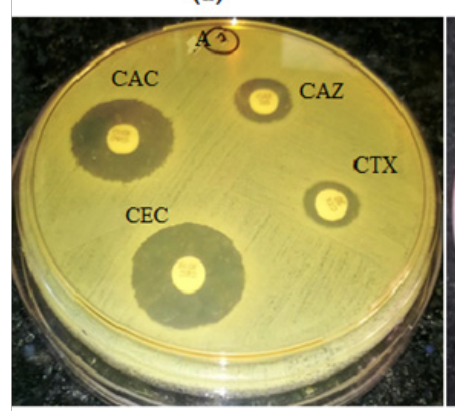

(c)

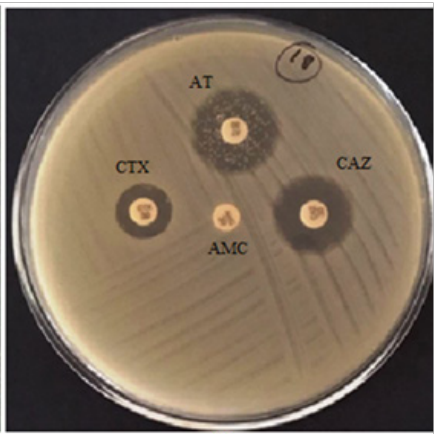

(b)

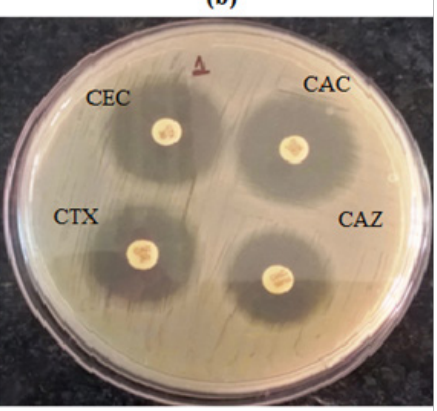

(d)
Figure 4 (a ,b, c and d) E. coli isolates showing Double disc synergy test and phenotypic confirmatory disc diffusion test (A) DDST positive E. coli isolate; (B) DDST negative E. coli isolate; (C) PCDDT positive $E$. coli isolate; (D) PCDDT negative $E$. coli isolate.

AMC, amoxicillin/clavunate;AT, azeotranum; CAC, ceftazidime/ clavulanic acid; CAZ, ceftazidime; CEC, cefotaxime/ clavulanic acid; CTX, cefotaxime

E-Test (Figure 5A-5D): ESBL positive $E$. coli isolates were further confirmed by E test ESBL strips. Regarding the results of E-strip test,
The MIC was interpreted as the point of intersection of the inhibition ellipse with the edge of the test. E-test strips for (MIX+/MIX) and $(\mathrm{CPM}+/ \mathrm{CPM})$ gave $\mathrm{MIC}$ value greater than 8 or not produce zone for MIX and CPM which indicated the presence of 27 ESBL E. coli.

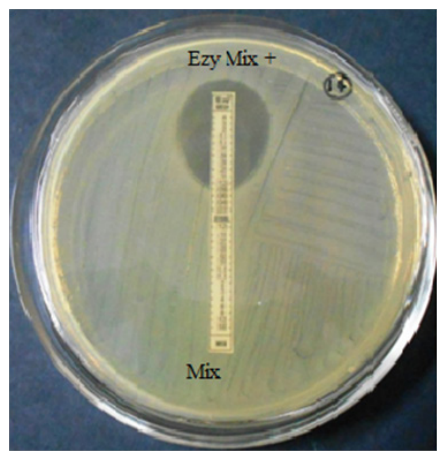

(a)

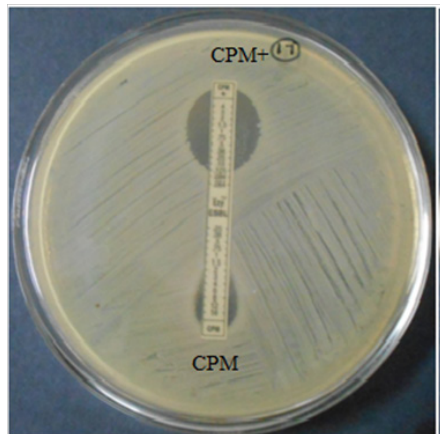

(c)

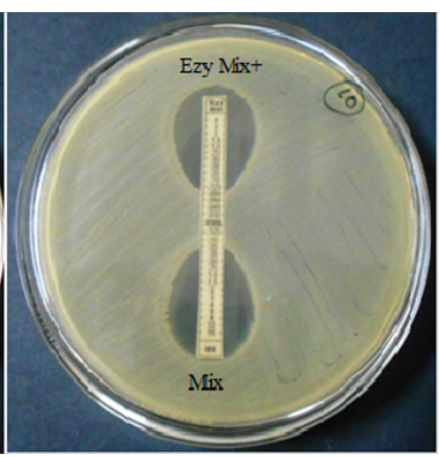

(b)

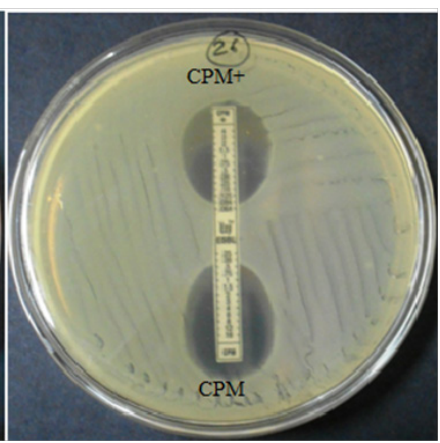

(d)

Figure 5 (A-D) E. coli isolates showing E-tests for ESBL confirmation

(A) E-test positive with $\mathrm{MIX}+/ \mathrm{MIX}$ strip; (B) E-test negative with $\mathrm{MIX+1}$ MIX strip; (C) E-test positive with CPM+/CPM strip; (D) E-test negative with CPM+/CPM strip

A significant association in prevalence of ESBL producer and ESBL non producer $E$. coli isolates was revealed $(\mathrm{P}<0.01)$ (Figure 6).

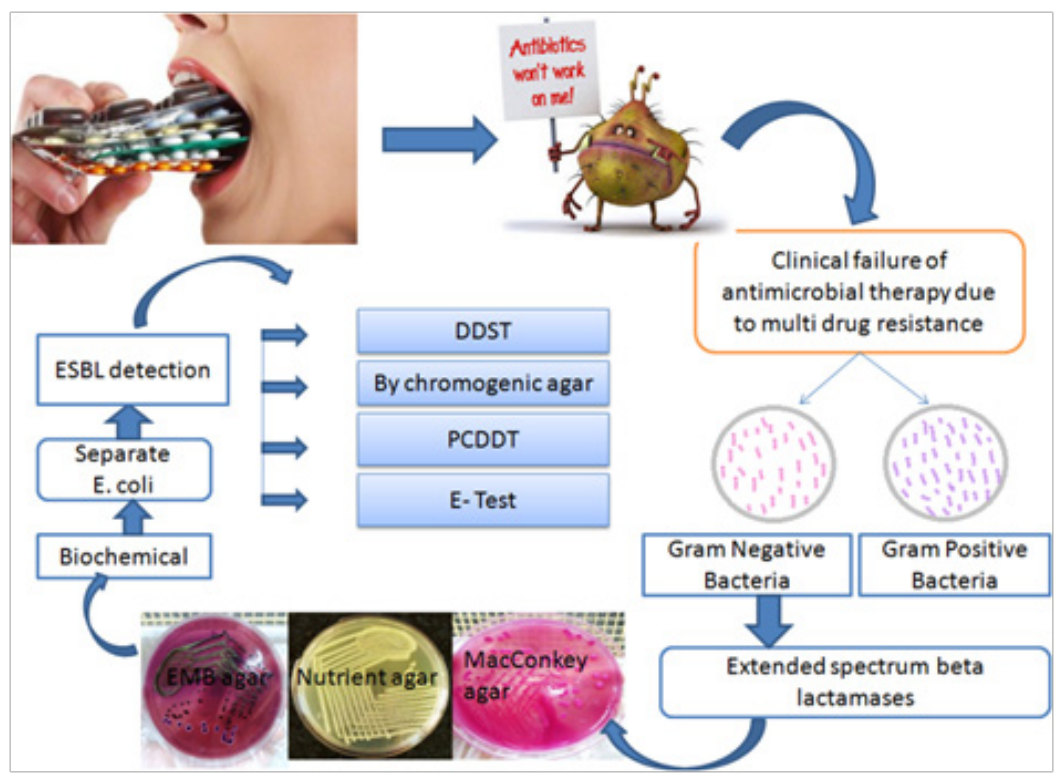

Figure 6 Graphical abstract.

Citation: Mishra A, Bist R, Sharma V. Study of pervasiveness, antimicrobial vulnerability and resolution of best method for determining extended spectrum beta lactamases Escherichia coli isolates. Hos Pal Med Int Jnl. 2019;3(2):59-64. DOI: 10.15406/hpmii.2019.03.00I52 


\section{Discussion}

ESBL producing family enterobacteriaceae are raising concern globally and currently a most important confronts to clinicians, communal health experts, and hospice infection control panel.

In the present study, we investigated ESBL producer $E$. coli isolates and susceptibility pattern towards a variety of antimicrobial drugs. Organisms producing ESBLs are clinically proven applicable cause of failure of cephalosporins therapy. ${ }^{28}$ ESBLs are mostly produced by with the aid of the family enterobacteriaceae, in $\mathrm{K}$. pneumoniae and E. coli. Bacteria harbouring ESBLs also acquire and most often exhibit supplementary resistances to other antimicrobial classes such as the floroquinolones, tetracyclines, co-trimoxazole, and aminoglycosides, its further restrict the therapeutic alternative and thus cause a therapeutic quandary. ${ }^{29}$

Current study revealed $37 \mathrm{E}$. coli isolates out of the 40 stool samples because $E$. coli bacterium is frequently found in gut of human and animals. Most of the strains of $E$. coli are part of beneficial bacterial flora in human gut, however a few are reported to cause infections in humans. ESBLs producing E. coli express resistivity to antibiotics used in treating infections.

Current investigation used different screening and confirmatory tests for the detection of ESBLs producing $E$. coli isolates and recorded $72.9 \%$ E. coli isolates with ESBLs production. Present study confirmed that PCDDT and E-test of ESBLs detection are most sensitive than DDST method. ${ }^{27,30}$ The high percentage of ESBLs producer E. coli was found. It may be due to overuse of antibiotics, prolonged hospital stay and long time exposure of antibiotics. ${ }^{31} \mathrm{~A}$ widespread resistance to third-generation cephalosporins among Enterobacterial E. coli was recorded..$^{32}$ ESBLs producer E. coli isolates were found resistant to amplicillin, piperacillin as well as extended-spectrum cephalosporin which is also in agreement according to the findings. ${ }^{33}$ ESBLs producers also showed resistivity to some other class of antibiotics, such as floroquinolones and cotromoxazole. ${ }^{34,35}$ Current study reported that Cabapenemase group of antibiotics are found highly susceptible to ESBLs producer as well as non ESBLs producer may be due to the stability and the high activity of carbapenem against most $\beta$-lactamases. Therefore, extended spectrum carbapenems are recommended to treat infections caused by ESBL producing bacteria. ${ }^{36}$ Present investigation reported the susceptibility rates to $\beta$-lactam inhibitor such as ampicillin/sulbactam and piperacillin/tazobactam in the ESBL E. coli $70.3 \%$ and $66.6 \%$, respectively, were higher than those to cefotaxime, ceftazidime, and cefepime because $\beta$ - lactam inhibitors be able to inhibit ESBL enzyme activity, and hydrolysis of bond. And also they were not highly use for treatment of community acquired infections. So they are considered an alternative group of drugs beside carbapenems to treat infections due to ESBL producing bacteria. ${ }^{37}$ Whereas, amoxicillin/clavunate $86.4 \%$ and amoxicillin/sulbactam $94.5 \%$ showed highly resistant to ESBLs as well as non ESBLs producer E. coli isolates because of their extreme uses.

High degree of susceptibility rate was observer to aminoglycosides drugs $94.5 \%$ for both ESBLs and non ESBLs producer E. coli isolates. Hence, in the group ESBL producers aminoglycosides may have contributed to the non-noteworthy findings in the medical and microbiological responses among the ESBL producers and nonESBL producers. Aminoglycosides contain excellent action adjacent to clinically important GNB. Aminoglycosides are main class of antibiotics which shows activity against many gram-negative rod shape bacilli. The enzymatic modification of antibiotic molecule is the common mechanism of aminoglycosides resistance. Aminoglycosides class show lesser resistance rates because they are injectable vaccines so restrictively used by community and health care settings. ${ }^{38}$

\section{Conclusion}

Findings of current research present the high prevalence of MDR ESBLs E. coli which imposes significant threat to public health. Surveillance antibiotic resistivity's of ESBL positive $E$. coli isolates ensure the careful use of antimicrobial drugs. Present study also concluded that PCDDT and E-test were best optimized methods for detection of ESBL producing organisms.

\section{Acknowledgments}

We would like to thank Prof. Aditya Shastri for help with managing our lab facilities on Banasthali University and running this study. We would also like to thank the Banasthali University, Rajasthan, India and Escort Fortis Hospital Rajasthan, India for providing lab facilities and samples, respectively.

\section{Conflicts of interest}

The authors declare there is no conflict of interest.

\section{References}

1. Dobias J, Denervaud-Tendon V, Poirel L, et al. Activity of the novel siderophore cephalosporin cefiderocol against multidrug-resistant Gramnegative pathogens. Eur J Clin Microbiol Infect Dis. 2017;36(12):2319 2327.

2. Pitout JD, Laupland KB. Extended spectrum beta lactamase producing Enterobacteriaceae: an emerging public-health concern. Lancet Infect Dis. 2008;8(3):159-166.

3. Alyamani EJ, Khiyami AM, Booq RY, et al. The occurrence of ESBLproducing Escherichia coli carrying aminoglycoside resistance genes in urinary tract infections in Saudi Arabia. Ann Clin Microbiol Antimicrob. 2017;16(1):1-13.

4. Mishra A, Sharma V, Bist R. Prevalence of extended spectrum $\beta$-Lactamases producing $E$. coli in conferring multi drug resistance against antimicrobials. Vegetos. 2018;31:96-101.

5. Ansari S, Nepal, HP, Gautam R, et al. Community acquired multi-drug resistant clinical isolates of Escherichia coli in a tertiary care center of Nepal. Antimicrob Resist Infect Control. 2015;4:1-8.

6. Ogbolu DO, Terry Alli OA, Webber MA, et al. CTX-M-15 is Established in most multidrug-resistant uropathogenic enterobacteriaceae and pseudomonaceae from hospitals in Nigeria. Eur $J$ Microbiology Immunol. 2018;8(1):20-24.

7. Igwe JC, Onaolapo JA, Kachallah M, et al. Molecular characterization of extended spectrum $\beta$-lactamase genes in clinical $E$. coli isolates. $J$ Biomed Sci Eng. 2014;7(5):276-285.

8. Harris PNA, Yin M, Jureen, R, et al. Comparable outcomes for $\beta$-lactam/ $\beta$-lactamase inhibitor combinations and carbapenems in definitive treatment of bloodstream infections caused by cefotaxime-resistant Escherichia coli or Klebsiella pneumonia. Antimicrob Resist Infect Control. 2015;4:1-10.

9. Stuart RL, Kotsanas D, Webb B, et al. Prevalence of antimicrobialresistant organisms in residential aged care facilities. Med J Aust. 2011;195(9):530-533.

10. Niumsup PR, Tansawai U, Na-udom A, et al. Prevalence and risk factors for intestinal carriage of CTX-M-type ESBLs in Enterobacteriaceae from a Thai community. Eur J Clin Microbiol Infect Dis. 2018;37(1):69-75. 
11. Cheikh A, Belefquih B, Chajai Y, et al. Enterobacteriaceae producing extended spectrum $\beta$-lactamases (ESBLs) colonization as a risk factor for developing ESBL infections in pediatric cardiac surgery patients: "retrospective cohort study". BMC Infect Dis. 2017;17(1):5-6.

12. Drawz SM, Papp-Wallace KM, Bonomo RA. New beta-lactamase inhibitors: a therapeutic renaissance in an MDR world. Antimicrob Agents Chemother. 2014;58(4):1835-1846.

13. Rawat D, Nair D. Extended-spectrum beta-lactamases in Gram Negative Bacteria. J Glob Infect Dis. 2010;2(3):263-274.

14. Livermore DM. Bacterial resistance: origins, epidemiology, and impact Clin Infect Dis. 2003;36(Suppl 1):S11-S23.

15. Onwuezobe IA, Orok FE. The bacterial isolates and plasmid profile of extended spectrum beta-lactamases producers causing urinary tract infection among pregnant women in Uyo, Nigeria. $J$ Biosci Med. $2015 ; 3: 25-30$

16. Yousefipour M, Rasoulinejad M, Hadadi A, et al. Bacteria producing extended spectrum $\beta$-lactamases (ESBLs) in hospitalized patients: prevalence, antimicrobial resistance pattern and its main determinants. Iran J Pathol. 2019;14(1):61-67.

17. Hawkey PM. Prevalence and clonality of extended-spectrum b-lactamases in Asia. Clin Microbiol Infect. 2008;14 Suppl 1:159-165.

18. Hijazi SM, Fawzi MA, Ali FM, et al. Prevalence and characterization of extended-spectrum beta-lactamases producing Enterobacteriaceae in healthy children and associated risk factors. Ann Clin Microbiol Antimicrob. 2016;15:1-9.

19. Abike TO, Olufunke OA, Temitope OO. Prevalence of extended spectrum $\beta$-lactamases in multidrug resistant strains of Gram-negative bacteria. Afr J of Microbiol Res. 2018;12(7):147-151.

20. Bourouis A, Moussa MB, Belhadj O. Multidrug-resistant phenotype and isolation of a Novel SHV- beta-Lactamase variant in a clinical isolate of Enterobacter cloacae. ऐ Biomed Sci. 2015;22(1):1-7.

21. Zhang H, Zhou Y, Guo S, et al. Prevalence and characteristics of extendedspectrum beta lactamase (ESBL)-producing Enterobacteriaceae isolated from rural well water in Taiwan, China. 2014. Environ Sci Pollut $R$ 2015;22(15):11488-11492.

22. Chander A, Shrestha CD. Prevalence of extended spectrum beta lactamase producing Escherichia coli and Klebsiella pneumoniae urinary isolates in a tertiary care hospital in Kathmandu, Nepal. BMC Res Notes. 2013;6:1-6.

23. Wayne PA. Performance standards for antimicrobial disk susceptibility tests. CLSI. 19th ed. 2012a; M02-A11.

24. Souverein D, Euser MS, Herpers LB, et al. Prevalence, risk factor and molecular epidemiology of highly resistant gram negative rods in hospitalized patients in the Dutch region Kennemerland. Antimicrob Resist Infect Control. 2016;5:2-10.

25. Wayne PA. Performance Standard for Antimicrobial Susceptibility Testing. CLSI. 2006; 16th Informational supplement: M100-S16.
26. Wayne PA. Performance standards for antimicrobial susceptibility testing. NCCLS. 2012; 12th informational supplement: M100-S12.

27. Khater ES, Sherif HW. Rapid detection of extended spectrum $\beta$-lactamase (ESBL) producing strain of Escherichia coli in urinary tract infections patients in Benha University Hospital, Egypt. Br Microbiol Res J. 2014;4(4):443-453.

28. Raji MA, Jamal W, Ojemeh O, et al. Sequence analysis of genes mediating extended-spectrum beta-lactamase (ESBL) production in isolates of Enterobacteriaceae in a Lagos Teaching Hospital, Nigeria. BMC Infect Dis. 2015;15:1-5.

29. Yadav KK, Adhikari N, Khadka R, et al. Multidrug resistant Enterobacteriaceae and extended spectrum $\beta$-lactamase producing Escherichia coli: a cross-sectional study in National Kidney Center, Nepal. Antimicrob Resist Infect Control. 2015;4:1-7.

30. Rampure R, Gangane R, Oli AK, et al. Prevalence of MDR-ESBL producing Klebsiella pneumoniae isolated from clinical Samples. J Microbiol Biotechnol Res. 2013;3(1):32-39.

31. Chaudhary U, Aggarwal R. Extended spectrum beta lactamases (ESBL) -An emerging threat to clinical therapeutics. Indian $J$ Med Microbiol. 2004;22(2):75-80

32. Vlieghe ER, Huang TD, Phe T, et al. Prevalence and distribution of beta-lactamase coding genes in third-generation cephalosporin-resistant Enterobacteriaceae from bloodstream infections in Cambodia. Eur J Clin Microbiol Infect Dis. 2015;34(6):1223-1229.

33. Anago E, Fanou LA, Akpovi CD, et al. Antibiotic resistance and genotype of beta-lactamase producing Escherichia coli in nosocomial infections in Cotonou, Benin. Ann Clin Microbiol Antimicrob. 2015;14:2-6.

34. Akram M, Shahid M, Khan AU. Etiology and antibiotic resistance patterns of community-acquired urinary tract infections in $\mathrm{J} \mathrm{N} \mathrm{M} \mathrm{C}$ Hospital Aligarh, India. Ann Clin Microbiol Antimicrob. 2017;6(4):1-7.

35. Manjunath GN, Prakash R, Vamseedhar A, et al. Changing trends in the spectrum of antimicrobial drug resistance pattern of uropathogens isolated from hospitals and community patients with urinary tract infections in Tumkur and Bangalore. IJBMR. 2011;2(2):504-507.

36. Han SB, Lee C, Lee SY, et al. Aminoglycoside therapy for childhood urinary tract infection due to extended spectrum $\beta$-lactamase-producing Escherichia coli or Klebsiella pneumonia . BMC Infect Dis. 2015;15:1-8.

37. Vardakas KZ, Tansarli GS, Rafailidis PI, et al. Carbapenems versus alternative antibiotics for the treatment of bacteraemia due to Enterobacteriaceae producing extended-spectrum beta-lactamases: a systematic review and meta-analysis. J Antimicrob Chemother. 2016;67(12):2793-2803.

38. Bajpai T, Pandey M, Varma M, et al. Prevalence of extended spectrum betalactamase producing uropathogens and their antibiotic resistance profile in patients visiting a tertiary care hospital in central India: Implications on empiric therapy. Indian J Pathol Microbiol. 2016;57(3):407-412. 